\title{
Pneumonia Associada à Ventilação Mecânica (PAVM) assistida pela quitosana
}

\author{
Ventilator-Associated Pneumonia (VAP) assisted by chitosan \\ Neumonía asociada a Ventilador (NAV) asistida por quitosano
}

Recebido: 04/02/2022 | Revisado: 10/02/2022 | Aceito: 13/02/2022 | Publicado: 20/02/2022

Luiza Aragão Paiva Pires Ferreira Mendes

ORCID: https://orcid.org/0000-0002-4017-6102

Centro Universitário Unifacid/Wyden, Brasil

E-mail: luizamendes0412@gmail.com

Amália Ramos de Sousa

ORCID: https://orcid.org/0000-0001-6485-4521

Centro Universitário Unifacid/Wyden, Brasil

E-mail:amaliaramossousa@gmail.com

Isabelle Vasconcelos Rodrigues

ORCID: https://orcid.org/0000-0003-2280-1020

Centro Universitário Unifacid/Wyden, Brasil

E-mail: isabellevasconcelosrodriguess@ hotimail.com

Jucilene da Silva Sousa

ORCID: https://orcid.org/0000-0001-9692-3499

Centro Universitário Unifacid/Wyden, Brasil

E-mail: sousajucilene9@gmail.com

Lara Isobel Vieira Bacelar

ORCID: https://orcid.org/0000-0003-1453-3652 Centro Universitário Unifacid/Wyden, Brasil

E-mail: laraisobel02@gmail.com

Fernando Henrique Lima Sa Machado

ORCID: https://orcid.org/0000-0001-5195-390X

Centro Universitário Unifacid/Wyden, Brasil

E-mail: biomed.fernando.machado@gmail.com

Alciene Pacheco da Silva

ORCID: https://orcid.org/0000-0003-4117-2792

Centro Universitário Unifacid/Wyden, Brasil

E-mail:alcienepacheco@ufpi.edu.br

Danielle Costa Lopes

ORCID: https://orcid.org/0000-0002-7382-1323

Universidade Federal do Piauí, Brasil

E-mail: dany197_@hotmail.com

Diego Rodrigues Pessoa

ORCID: https://orcid.org/0000-0002-8981-673X

Universidade Federal do Piauí, Brasil

E-mail: fisio.diegorodrigues@gmail.com

Nágila Iane Pacheco

ORCID: https://orcid.org/0000-0002-2836-1639

Centro Universitário Unifacid/Wyden, Brasil

E-mail: nagilaiane@hotmail.com

\begin{abstract}
Resumo
Introdução: A pneumonia associada à ventilação mecânica (PAVM) é uma das preponderantes causas das infecções nosocomiais em UTIs que ocasionando elevação do tempo de internação, despesas médicas, crescimentos nos índices de morbilidade e mortalidade, com o aumento de bactérias resistentes aos antibióticos, corrobora na aplicação de novos biomateriais, com isso, a quitosana é estudada por constituir por diversas propriedades funcionais, como antimicrobiana. Metodologia: A pesquisa realizada é uma revisão sistemática através da consulta em bancos de dados online para o levantamento das informações. As principais bases de dados de periódicos utilizados para as buscas ativas dos artigos foram: ANVISA, OMS, PUBMED, Science Direct, SCIELO. Resultados e discussões: A interação microbiana dentro do biofilme colabora para a PAVM, nesse sentido ocorre uma implicação na terapia antimicrobiana, entretanto a resistência aos fármacos utilizados revalida a necessidade de se encontrar novos agentes antimicrobianos, a quitosana e os seus derivados possuem versatilidade, baixo custo e vasto espectro de atividade antimicrobiana contra bactérias gram-positivas, gram-negativas, fungos filamentosos e leveduras. Conclusão: verificase que uma forma provável de combater as infecções bacterianas relacionadas a ventilação mecânica é a quitosana e seus derivados, que possuem atividades antimicrobianas relevantes e grande potencial para utilização dos mesmos no combate a tenacidade de microorganismos.
\end{abstract}

Palavras-chave: Ventilação mecânica; Pneumonia; Quitosana; Antibacteriano; Biofilme. 


\begin{abstract}
Introduction: Ventilator-associated pneumonia (VAP) is one of the main causes of nosocomial infections in ICUs, which leads to an increase in length of stay, medical expenses, increases in morbidity and mortality rates, with an increase in antibiotic-resistant bacteria, corroborates in the application of new biomaterials, therefore, chitosan is studied because it has several functional properties, such as antimicrobial. Methodology: The research carried out is a systematic review by consulting online databases to collect information. The main journal databases used for active article searches were: ANVISA, WHO, PUBMED, Science Direct, SCIELO. Results and discussions: The microbial interaction within the biofilm contributes to the PAVM, in this sense there is an implication in antimicrobial therapy, however the resistance to the drugs used validates the need to find new antimicrobial agents, chitosan and its derivatives have versatility, low cost and broad spectrum of antimicrobial activity against gram-positive and gramnegative bacteria, filamentous fungi and yeasts. Conclusion: it appears that a probable way to combat bacterial infections related to mechanical ventilation is chitosan and its derivatives, they have relevant antimicrobial activities and great potential for their use in combating the tenacity of microorganisms.
\end{abstract}

Keywords: Mechanical ventilation; Pneumonia; Chitosan; Anti-bacterial; Biofilm.

\title{
Resumen
}

Introducción: La neumonía asociada a ventilador (NAV) es una de las principales causas de infecciones nosocomiales en las UCI, lo que conduce a un aumento de la estancia hospitalaria, gastos médicos, aumento de las tasas de morbimortalidad, con aumento de bacterias resistentes a los antibióticos, corrobora en la aplicación de nuevos biomateriales, con esto se estudia el quitosano debido a que tiene varias propiedades funcionales, como antimicrobiano. Metodología: La investigación realizada es una revisión sistemática mediante la consulta de bases de datos en línea para recopilar información. Las principales bases de datos de revistas utilizadas para la búsqueda activa de artículos fueron: ANVISA, OMS, PUBMED, Science Direct, SCIELO. Resultados y discusiones: La interacción microbiana dentro del biofilm contribuye a la PAVM, en este sentido existe una implicación en la terapia antimicrobiana, sin embargo la resistencia a los fármacos utilizados valida la necesidad de encontrar nuevos agentes antimicrobianos, el quitosano y sus derivados tienen versatilidad, baja costo y amplio espectro de actividad antimicrobiana contra bacterias grampositivas y gramnegativas, hongos filamentosos y levaduras. Conclusión: parece que una forma probable de combatir las infecciones bacterianas relacionadas con la ventilación mecánica es el quitosano y sus derivados, tienen actividades antimicrobianas relevantes y un gran potencial para su uso en el combate a la tenacidad de los microorganismos.

Palabras clave: Ventilacion mecanica; Neumonía; Quitosano; Antibacteriano; Biopelícula.

\section{Introdução}

Os pacientes que apresentam insuficiência respiratória - aguda ou crônica - é direcionado para utilizar a ventilação Os pacientes que apresentam insuficiência respiratória - aguda ou crônica - é direcionado para utilizar a ventilação mecânica (VM), parcial ou total, como suporte para compensar a ventilação espontânea, usualmente em unidades de terapia intensiva (UTI) (Silva et al.,2020; Santos et al.,2020; Pinto et al.,2021). Refere-se à inserção de forma invasiva com um tubo endotraqueal, com a finalidade de garantir as trocas gasosas, precaver a fadiga dos músculos da respiração, reduzir a consumação de $\mathrm{O}^{2}$ e obter conforto respiratório (Lima et al., 2017; Santos, et al., 2020; Silva et al., 2020).

A intubação endotraqual acarreta a elevação dos riscos de infecção do trato respiratório que ocorre, cerca de 48 horas após o procedimento, atingindo de 5 a 40\% dos eivados (Karakuzu et al., 2018; Vazquez \& Kollef, 2018; Soussan et al., 2018; Mohd et al., 2019; suaya et al., 2020; Papazian, Klompas, Luyt, 2020; Pinto et al., 2021). A pneumonia associada à ventilação mecânica (PAVM) é uma das preponderantes causas das infecções nosocomiais em UTIs que ocasionando elevação do tempo de internação do paciente com a utilização de antibióticos, despesas médicas, crescimentos nos índices de morbilidade e mortalidade em todos os países (Soussan et al., 2018; Vazquez \& Kollef, 2018; Karakuzu et al., 2018; Moah et al., 2019; Silva et al.,2020; Papazian, Klompas, Luyt, 2020; Pinto et al., 2021).

Diversos patógenos são encarregados por promover a pneumonia (Gram positivos e/ou negativos), principalmente Streptococcus pneumoniae, porém quando relacionados à PAVM, não é habitual (Suaya et al., 2020). Ao diagnosticar em seu início precoce - até quatro dias de intubação - são, comumente, encontrados Klebsiella pneumoniae, Staphylococcus aureus resistente à meticilina (MRSA) e Haemophilus influenzae. No caso da diagnose tardia - após os quatros dias de 
intubação- são detectadas bactérias oportunistas, como Acinetobacter spp, MRSA e Pseudomonas aeruginosa (Lima et al., 2017; Souza et al., 2017; Karakuzu et al., 2018; Papazian et al., 2020; Suaya et al., 2020; Papazian et al.,2020).

A incidência é relativa de acordo com a população pesquisada, os acometidos, geralmente, são traumáticos, como contusão pulmonar ou lesão cerebral, câncer, doenças obstrutivas pulmonares, síndrome do desconforto respiratório agudo (SDRA) (Papazian et al., 2020). Nas UTIs, os enfermos manifestam-se uma precária higiene bucal e com o tubo traqueal, favorecem a formação de biofilmes (Souza et al., 2017, Lima et al., 2017). Com a proliferação do desse, os microorganismos tornam-se resistentes as terapias medicamentosas e elevam a taxa de morbimortalidade, logo opera como barreira para impossibilitar a penetração do fármaco e do organismo reconhecer o invasor (Lima et al., 2017). Ademais, com a aplicação de antibióticos exacerbadamente, demonstra efeitos colaterais e propicia a resistência (Lamiyan et al., 2020).

A excedência resistência bacteriana empregada pela abrangência de fármacos de amplo espectro e implantes. Corrobora para investigação de novos antimicrobianos, preferencialmente em fontes naturais, tornando-se um dos principais meios de pesquisa por seus diversos efeitos farmacológicos, por sua eficácia duradoura, segurança, confiabilidade e mínimos efeitos adversos (Sidrim et al., 2019)

Uma dessas fontes naturais é o biopolímero quitina, indentificado, principalmente, na parede celular dos fungos e no exoesqueleto de crustáceos. Todavia, geralmente sua forma desacetilada é empregada, denominada quitosana (CS) (Mohan et al., 2021). A CS é um polímero natural, atóxico, biocompatível, biodegradável e policatiônico natural com atividades antimicrobianas (Naveed et al., 2019). Utilizado na produção de biomateriais, com o intuito de reduzir as resistências bacterianas. Além disso, devido a sua capacidade mucoadesiva inerente e do seu poder de penetração do biofilme, é avaliada como um transmissor de potência elevada para a liberação da droga para os pulmões, fomentando assim as suas atribuições e evidenciando a eficiência (Kucukogluet al., 2019; Uzuneret et al., 2019; Kenaret al., 2019, Karadenizli et al., 2019). Outrossim, as produções antibacterianas in vitro, juntamente com os resultados propostos pela citotoxicidade de micropartículas da CS favorecem diversas vantagens, como proporcionar uma menor nanotoxicidade e depuração local (Kucukogluet al., 2019).

Dessa forma, com o aumento de bactérias resistentes aos antibióticos, corrobora na aplicação de novos biomateriais inerentes são extremamente necessários para combater e diminuir o número de casos agravados decorrentes a isso. Com isso, a quitosana é estudada por constituir por diversas propriedades funcionais, como antimicrobiana, proporcionando uma nova alternativa (Huang et al., 2021). Com isso, a finalidade da pesquisa proposta é analisar a quitosana, por bancos de dados secundários, na assistência da pneumonia associada à ventilação mecânica (PAVM).

\section{Metodologia}

A pesquisa realizada é uma revisão sistemática (Fernando et al., 2020), sobre "Pneumonia Associada à Ventilação Mecânica (PAVM) assistida pela quitosana", através da consulta em bancos de dados online para o levantamento das informações. As principais bases de dados de periódicos utilizados para as buscas ativas dos artigos foram: Agência Nacional de Vigilância Sanitária (ANVISA), Organização Mundial da Saúde (OMS), PUBMED, Science Direct, Scientific Electronic Library Online (SCIELO).

Os critérios de inclusão sobre o referido levantamento deram-se através do uso das combinações de palavras-chaves em inglês "Mechanical ventilation, biofilm, antibacterial, chitosan, pneumonia", e as palavras em português "Ventilação mecânica, biofilm, antibacteriana, quitosana, pneumonia”. Com o intuito de tornar a busca mais específica, realizou-se as conexões entre os termos com a utilização dos operadores booleanos "AND” e "NOT”, que na íntegra retrata-se da temática referente de analisar a quitosana, por bancos de dados secundários, na assistência da pneumonia associada à ventilação mecânica. Como critério de exclusão elimiram-se 1334 artigos de 1365 encontrados, limitando-se os resultados das buscas em 
inglês, espanhol e português, em seguida das compilações resolveu-se as divergências por consenso em relação à duplicidade, as não relacionadas à temática e as aglutinadas com outras substâncias, como óleos e antibióticos.

Neste estudo, as referências bibliográficas selecionadas e utilizadas na pesquisa foram publicadas e indexadas nos referidos bancos de dados dos anos de 2017 a 2022. Neste levantamento são reportados 1 da ANVISA, 1 da OMS, 9 da PUBMED, 11 do Science Direct, 9 da SCIELO como demonstra-se no quadro a seguir (Figura 1). Utilizou-se o total de 31 artigos, todas as pesquisas, como a triagem de títulos, resumos e a seleção de estudos executaram-se pelos pesquisadores que trabalharam de forma independente. Assim, utilizaram-se todos os artigos considerados potencialmente legíveis para revisão do seu texto completo. Após isso, para a análise dos dados utilizou-se o programa Microsoft Excel versão Windows 10.

Figura 1: Fluxograma com as etapas de análise realizadas para seleção dos artigos científicos.

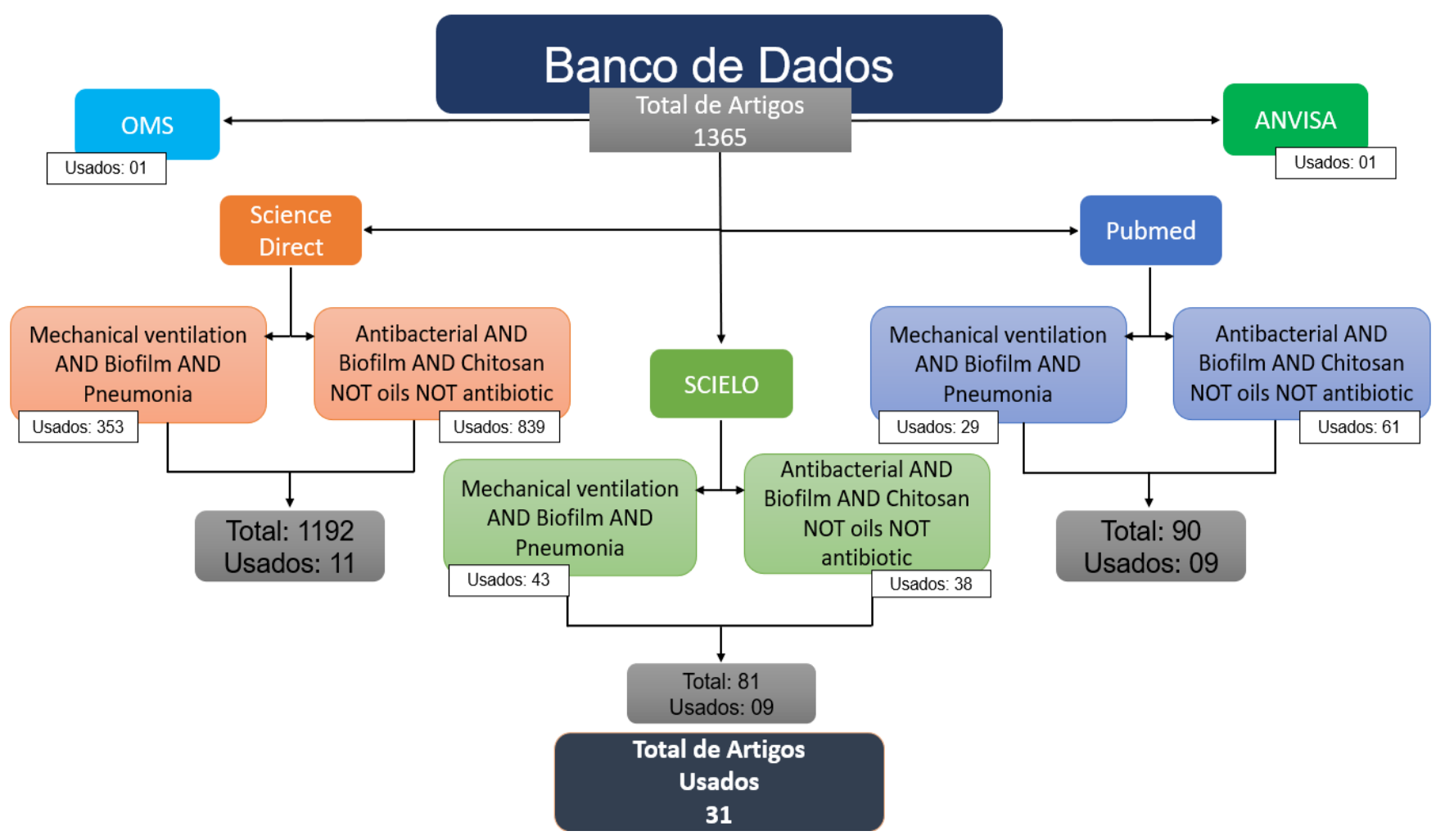

Fonte: Sousa (2021).

A Quadro 1 apresenta a listagem das principais publicações encontradas na busca ativa nos bancos de dados de periódicos, acerca da temática "Pneumonia Associada à Ventilação Mecânica (PAVM) assistida pela quitosana”. 
Quadro 1: Artigos científicos listados dos bancos de dados.

\begin{tabular}{|c|c|c|}
\hline Título do artigo & Autores e ano da pesquisa & $\begin{array}{c}\text { Revista } \\
\text { cientifica }\end{array}$ \\
\hline Critérios diagnósticos de infecção relacionada à assistência à saúde & ANVISA, 2017 & ANVISA \\
\hline A review on chitosan and its nanocomposites in drug delivery & Ali; Ahmed,2018 & PUBMED \\
\hline Is Zero Ventilator-Associated Pneumonia Achievable? & Vazquez ; Kollef, 2018 & PUBMED \\
\hline $\begin{array}{l}\text { Biomedical application of chitosan-based nanoscale delivery systems: Potential usefulness } \\
\text { in siRNA delivery for cancer therapy }\end{array}$ & Ashrafizadeh et al., 2021 & PUBMED \\
\hline The challenge of ventilator-associated pneumonia diagnosis in COVID-19 patients & François et al., 2020 & PUBMED \\
\hline Ventilator-associated pneumonia in adults: a narrative review. & Papazian; Klompas; Luyt, 2020 & PUBMED \\
\hline $\begin{array}{l}\text { Bioinspired Heteromultivalent Chitosan- } \alpha-\mathrm{Fe}_{2} \mathrm{O}_{3} / \text { Gadofullerene Hybrid Composite for } \\
\text { Enhanced Antibiotic-Resistant Bacterial Pneumonia }\end{array}$ & Huang et al., 2021 & PUBMED \\
\hline $\begin{array}{l}\text { Diagnosis of ventilator-associated pneumonia in critically ill adult patients-a systematic } \\
\text { review and meta-analysis }\end{array}$ & Fernando et al., 2019 & PUBMED \\
\hline $\begin{array}{l}\text { Identification of Streptococcus pneumoniae in Hospital-acquired Pneumonia in Adults: A } \\
\text { Systematic Review }\end{array}$ & Suaya et al., 2020 & PUBMED \\
\hline $\begin{array}{l}\text { Chlorpromazine-impregnated catheters as a potential strategy to control biofilm-associated } \\
\text { urinary tract infections }\end{array}$ & Sidrim et al., 2019 & PUBMED \\
\hline $\begin{array}{l}\text { Chitosans as new tools against biofilms formation on the surface of silicone urinary } \\
\text { catheters }\end{array}$ & Campana et al., 2018 & Science direct \\
\hline Chitosan as an effective inhibitor of multidrug resistant Acinetobacter baumannii & Costa et al., 2017 & Science direct \\
\hline Ventilator-associated pneumonia: The central role of transcolonization & Soussan et al., 2018 & Science direct \\
\hline $\begin{array}{l}\text { The global prevalence of multidrug-resistance among Acinetobacter baumannii causing } \\
\text { hospital-acquired and ventilator-associated pneumonia and its associated mortality: A } \\
\text { systematic review and meta-analysis. }\end{array}$ & Sazlly et al., 2019 & Science direct \\
\hline Chitosan oligosaccharide (COS): An overview & Naveed et al., 2019 & Science direct \\
\hline $\begin{array}{l}\text { The global prevalence of multidrug-resistance among Acinetobacter baumannii causing } \\
\text { hospital-acquired and ventilator-associated pneumonia and its associated mortality: A } \\
\text { systematic review and meta-analysis }\end{array}$ & Mohd et al., 2019 & Science direct \\
\hline A study on structural comparisons of $\alpha$-chitin extracted from marine crustacean shell waste & Mohan et al., 2021 & Science direct \\
\hline $\begin{array}{l}\text { Recent advancements in applications of chitosan-based biomaterials for skin tissue } \\
\text { engineering. }\end{array}$ & Madni et al., 2021 & Science direct \\
\hline $\begin{array}{l}\text { Investigating the potential of endolysin loaded chitosan nanoparticles in the treatment of } \\
\text { pneumococcal pneumonia }\end{array}$ & Gondil; Harjai; Chhibber, 2021 & Science direct \\
\hline $\begin{array}{l}\text { Preparation of chitosan nanoparticles by TPP ionic gelationcombined with spray drying, } \\
\text { and the antibacterial activity of chitosan nanoparticles and a chitosan nanoparticle-- } \\
\text { amoxicillin complex. }\end{array}$ & Tan et al., 2017 & Science direct \\
\hline Fatores de risco de pneumonia associada ao ventilador em pacientes criticamente III & Wu et al., 2019 & Science direct \\
\hline Chitosan and their derivatives: Antibiofilm drugs against pathogenic bacteria & Khan et al., 2020 & Science direct \\
\hline $\begin{array}{l}\text { In vitro antibacterial activity of ciprofloxacin loaded chitosan microparticles and their } \\
\text { effects on human lung epithelial cells }\end{array}$ & Kucukoglu et al., 2019 & Science direct \\
\hline
\end{tabular}




\begin{tabular}{|c|c|c|}
\hline Nomear a doença coronavírus (COVID-19) e o vírus que a causa. & OMS, 2020 & OMS \\
\hline Fatores de risco prognóstico na pneumonia associada ao ventilador. & Karakuzu et al., 2018 & SCIELO \\
\hline $\begin{array}{l}\text { Venom peptides in association with standard drugs: a novel strategy for combating } \\
\text { antibiotic resistance-an overview. }\end{array}$ & Lamiyan; Dalal; Kumar, 2020 & SCIELO \\
\hline $\begin{array}{l}\text { Characteristics of chitosan fiber and their effects towards improvement of antibacterial } \\
\text { activity }\end{array}$ & Li et al., 2022 & SCIELO \\
\hline $\begin{array}{l}\text { Analysis of biofilm production by clinical isolates of Pseudomonas aeruginosa from } \\
\text { patients with ventilator-associated pneumonia. }\end{array}$ & Lima et al., 2017 & SCIELO \\
\hline $\begin{array}{l}\text { Efficiency of different protocols for oral hygiene combined with the use of chlorhexidine in } \\
\text { the prevention of ventilator-associated pneumonia }\end{array}$ & et al., 2021 & SCIELO \\
\hline $\begin{array}{l}\text { Good nursing practices towards patients on invasive mechanical ventilation in hospital } \\
\text { emergencya. }\end{array}$ & Santos et al., 2020 & SCIELO \\
\hline $\begin{array}{l}\text { Impact of oral hygiene in patients undergoing mechanical ventilation in the COVID-19 } \\
\text { pandemic. }\end{array}$ & Silva et al., 2020 & SCIELO \\
\hline $\begin{array}{l}\text { Combinação de escovação dentária e clorexidina em comparação com o uso exclusivo de } \\
\text { clorexidina para reduzir o risco de pneumonia associada à ventilação: Uma revisão } \\
\text { sistemática com meta-análise }\end{array}$ & Silva et al., 2021 & SCIELO \\
\hline $\begin{array}{l}\text { Association between pathogens from tracheal aspirate and oral biofilm of patients on } \\
\text { mechanical ventilation }\end{array}$ & Souza et al., 2017 & SCIELO \\
\hline
\end{tabular}

Fonte: Mendes (2021).

\section{Resultados e Discussão}

\subsection{Pneumonia associada à Ventilação Mecânica}

Os pacientes com fraqueza respiratória e metabólica usufruem da ventilação mecânica (VM), método de ventilação artificial que assegura a manutenção das trocas gasosas essenciais ao organismo e é apontado como um suporte terapêutico, habitualmente, aplicado em unidades de terapia intensiva (UTI). Todavia, expõe os enfermos ao perigo de adquirir Pneumonia Associada à Ventilação Mecânica (PAVM) (Lima et al., 2017).

Inúmeros fatores de risco são respectivos, como aplicação prolongada da ventilação mecânica, pessoas de gênero masculino, senis, apresentadores de comorbidades (cadiopulmonar), aspiração, regurgitação, cirurgias invasivas, queimaduras, lesão ou inflamação no pulmão, disfunção imunológica sistêmica, fatores genéticos, indiscriminação de antibioticoterapia prévia, entre outras (Silva et al., 2021; Wu et al., 2019). A queimadura é um fator de risco de PAV por inflamação pulmonar consequente de lesão pulmonar direta ou disfunção imunológica sistêmica, polimorfismos genéticos relacionados a mediadores inflamatórios é capaz de ampliar o risco do seu progresso, eventualmente devido a uma resposta ineficiente às bactérias (Silva et al., 2021).

A VM é um método de reforço no tratamento de enfermo com insuficiência respiratória crônica aguda ou acentuada. Os objetivos essenciais são: aliviar a tensão dos músculos respiratórios, manterem as trocas gasosas, regressar ou precaver a exaustão dos músculos respiratórios, reduzirem o consumo de oxigênio e amenizar o desconforto respiratório (Silva et al., 2020). A taxa de mortalidade de PAV comumente varia entre 25\% a 50\%. Apesar disso, é capaz de elevar para $70 \%$, em alguns casos. Conforme os dados do Sistema Nacional de Vigilância de Infecções Nosocomiais (NNIS), em torno de 2,4-14,7 dos casos de pneumonia se manifestam em 1000 dias de VM (Kaeakuzu et al., 2018).

A PAVM é uma importante Infecção Pertencente à Saúde (HCRI) e provoca o acréscimo na mortalidade, dias de internação na UTI e custos hospitalares, essa sucede mediante quatro mecanismos, que são: (1) aspiração de secreções, (2) 
inalação de aerossóis contaminados, (3) disseminação de bactérias por via hematogênica e (4) translocação de bactérias do trato gastrointestinal. Dentre esses mecanismos, a aspiração de secreção da cavidade oral e orofaringe é o mais pertinente, estando designada a pneumonia aspirativa (Silva et al., 2020).

A detecção prévia de PAV é crítica, devido à administração tardia do recurso terapêutico, estando correlacionada ao acréscimo da mortalidade. Todavia, a relevância da ministração rápida para o tratamento, é preciso balancear contra os perigos de antibióticos dispensáveis, contendo resistência aos tais e superinfecções, sobretudo na UTI. Encontrar o equilíbrio certo é um desafio, visto que, é difícil a sua diagnose, por não existir um padrão de referência prático, as taxas e resultados constatados de PAV diversificam vastamente, conforme o conteúdo empregue e dois terços dos pacientes tratados, estando ou não eivados (Fernando et al., 2020).

A diagnose da pneumonia é complexa, as três partes indispensáveis para a constatação de PAV em concordância com os padrões atuais são sinais e sintomas, radiografia de tórax, e exames laboratoriais. No presente momento, não existe um padrão ouro para o seu diagnóstico, além disso, majoritariamente as demarcações empreguem não contém sensibilidade ou particularidade suficiente para determinar com exatidão (Agência Nacional de Vigilância Sanitária- ANVISA, 2017). Os dados microbiológicos são manuseados na tentativa de aperfeiçoar a exatidão do diagnóstico em razão da baixa especificidade dos padrões clínicos esporadicamente (Lima et al., 2017).

Os médicos habitualmente expõem com indicadores radiográficos, clínicos e laboratoriais para diagnosticar PAV e iniciar o tratamento empírico. Compreende-se em secreções purulentas, febre, hipoxemia, contagem elevada de leucócitos, infiltrado radiográfico de tórax novo ou gradativo e culturas positivas de aspirado endotraqueal (ETA) ou técnicas de amostragem broncoscópica (lavagem broncoalveolar [BAL] e escova de espécime protegida [PSB]) (Fernando et al., 2020).

Os métodos de precaução para PAV abrangem intercessão como erguer a cabeça do enfermo, aplicação de medicamentos profilaticamente, definirem o período da VM e impedir a sedação. A higiene é indicada como um encargo principal da prevenção e, com aplicação padronizada, consegue reduzir substancialmente a índice de infecções do trato respiratório através da colonização microbiana (Silva et al., 2020; Pinto et al., 2021).

\subsection{Resistência Bacteriana}

Os antibióticos são as entidades químicas que erradicam as bactérias ou tardam sua evolução. Porém, estes fármacos milagrosos desde o desenvolvimento da penicilina, não deixavam de manifestar efeitos colaterais graves. Estabeleceu-se que a aplicabilidade em longo prazo de forma demasiada deu-se origem a uma desordem séria denominada como resistência antimicrobiana. Os agentes apresentam técnicas para combater com sucesso por intermédio de distintos mecanismos, integrando alteração em seus genes, uma opção de sobrevivência aceita por microrganismos patogênicos e não patogênicos. Os altos níveis de uso regular e acentuado de antibióticos comerciais dificultam a conjectura e prejudica a efetividade dos medicamentos produzidos pela indústria farmacêutica (Lamiyan et al., 2020).

Propõe-se que, o tubo traqueal atue como um gatilho para PAV pela produção de biofilme em sua superfície, contribuindo com a patogênese da infecção. A interação microbiana dentro do biofilme colabora para a patologia, nesse sentido ocorre uma implicação na terapia antimicrobiana, ampliando as taxas de morbimortalidade associadas a esta infecção. O decréscimo para a produção da placa bacteriana no tubo endotraqueal durante a VM e, em sequência, diminuir a frequência da PAV, a purificação da microbiota oral e a redução das placas dentais tem sido aplicada, posto que ambas sejam fontes potenciais para a sua presença (Lima et al., 2017).

O biofilme é composto por comunidades bacterianas inseridas na matriz polissacarídica extracelular (EPS). Contêm uma estrutura tridimensional que fornece às células bacterianas uma matriz de crescimento e um estado de sobrevivência estável, protege as células das condições ambientais adversas e conserte a comunicação entre essas, através do quorum sensing 
(QS), que contêm papel primordial na elaboração do composto e é alvo dos pesquisadores na procura por métodos contra infecções de $P$. aeruginosa (Neves et al., 2021).

Essa matriz é um reservatório permanente de microrganismos que causam infecções em áreas afastadas do corpo. Os patógenos respiratórios, regularmente, não são detectados na microbiota oral de individuo saudáveis, mas pacientes hospitalizados são suscetíveis à colonização por estes microrganismos. Outrossim, os internados em UTIs, continuamente, apontam higiene bucal precária, com a adição considerável da camada protetora. Além disso, esses elementos impossibilitam a inserção de agentes quimioterápicos dificultando sua eliminação (Souza et al., 2017).

A indagação da resistência aos antibióticos influenciou a análise de novos fármacos alternativos para o monitoramento de infecções bacterianas com efeitos sinérgicos. Desde os tempos antigos, o homem usufrui de materiais naturais para terapias medicamentosas. Mediante o abrupto acréscimo da resistência, os empenhos científicos processados são redirecionados para a pesquisa de possibilidades da natureza que sejam possantes, porém limitando sua toxicidade (Lamiyan et al., 2020). Um elevado predomínio de patógenos respiratórios, como Acinetobacter ssp e Pseudomonas spp., é possível detectar na saliva e no biofilme dentário de hospitalizados. Níveis consideráveis de Streptococcus pneumoniae, Klebsiella pneumoniae, Staphylococcus aureus e Enterobacter cloacae, também são avistados em pacientes de UTI (Souza et al., 2017; Suaya et al., 2020).

Na atualidade, a P. aeruginosa é exposta como fundamental agente de PAV em UTI, ocasionando em torno de 50\% dos casos de PAV. Essa infecção microbiana ocorre, preponderantemente, em pacientes críticos e imunocomprometidos e são referentes ao aumento da morbimortalidade nas enfermarias e sua falta de susceptibilidade ao tratamento (Lima et al., 2017).

No caso da Acinetobacter baumannii é inerentemente excessivamente resistente a diversos fármacos, tornando um significativo causador de infecções nosocomiais, particularmente na UTIs. A prevalência geral de multirresistência entre A. baumannii promovendo pneumonia adquirida em hospitais (HAP) e PAV agrupada em 114 estudos deu-se 79,9\% (IC de 95\% 73,9-85,4\%). As regiões primordiais com predominâncias exposta são América Central (100\%), América Latina e Caribe (100\%) e Europa Ocidental (91,4\%; IC de 95\%, 70-99,9\%) (Sazlly et al., 2019).

Apesar de que o Streptococcus pneumoniae seja a causa bacteriana mais comum de enfermidades pneumonia adquirida na comunidade (PAC), seu papel na HAP não é claro. Os microrganismos constantemente indicados em HAP ou PAV são Pseudomonas aeruginosa, Staphylococcus aureus, Klebsiella spp., Escherichia coli, Enterobacter spp., e Acinetobacter spp., que aglutinadas acarretam por volta de $80 \%$ dos episódios. A PAV de início precoce é geralmente ocasionada por antibióticos, bactérias sensíveis, relativa à comunidade, como Haemophilus spp, mas sensíveis à meticilina $S$. aureus (MSSA), enquanto a VAP de início atardado é usualmente propiciada por multirresistente patógenos associados à exposição ao ambiente de saúde, incluindo $P$. aeruginosa, espécies de Acinetobacter spp. e $S$. aureus resistente à meticilina (MRSA) (Suaya et al., 2020).

\subsection{Quitosana}

A quitina, incluso com seus derivados, é um biomaterial, está gradativamente mais popularizado, devido a várias características vantajosas. Esse é o principal componente dos exoesqueletos dos artrópodes e igualmente, encontrado nas paredes celulares dos fungos, casulos de mariposas, diatomáceas, algas coralina, moluscos, protistas, poliquetas, dentro de peixes e esponjas de água doce. Considerado o segundo biopolímero com maior abundancia, depois da celulose. A quitina é facilmente processada em seus derivados, como a quitosana. Este demonstra as suas aplicações promissoras em uma ampla gama de áreas, incluindo ciência de alimentos, medicina, agricultura e o campo biomédico (Casteleijn et al., 2018).

Este biomaterial é constituído por cadeias lineares catiônicas com grupos amino, nos quais os grupos radicais livres lhes conferem grande potencial para reagir com uma grande variedade de moléculas. Assim, este biopolímero ganha à 
disponibilidade para se ligar a grupos pendentes (Madni et al., 2021). Além disso, sulfatando grupos amina, as reversões das características da CS obtidas com a insolubilidade em pH alto e suas propriedades hemostáticas e catiônicas. Consiste em inserir especificidades anticoagulantes no processo de sulfatação, com isso, as moléculas serão aniônicas e solúveis em água. Logo, os grupos anexados a quitosana permitem a fabricação de materiais versáteis com funcionalidade única e alterando e/ou as propriedades físicas desses materiais (Kalantari et al.,2019).

A quitosana (CS), extraída da quitina através de processos químicos, como no caso de desacetilação por hidrólise alcalina e posterior tratamento com soluções ácidas, formada por 2-amino-2 desoxi-D-glucopiranose ligada por $\beta(1 \rightarrow 4)$ glicosídico. Outro método é a desacetilação termoquímica da quitina em meio alcalino, naturalmente captada pelo uso de fungos. Dentre essas metodologias alcalinas é usualmente aplicados, como hidrólise em posição acetilada executando com soluções de $\mathrm{NaOH}$, levando à $\mathrm{N}$-desacetilação do polímero e o sulfato de hidrazina e hidrazina anidra são outras técnicas complementares (Kalantari et al.,2019).

Nos últimos anos, esse biopolímero se introduziu no domínio médico devido às suas propriedades intrínsecas atraentes, nomeadamente a elevada biocompatibilidade, atóxicidade, a biodegradabilidade, fungistática, a baixa alergenicidade, a limitada citotoxicidade nas células humanas, hemostasia. Outroassim, a CS e os seus derivados possuem um vasto espectro de atividade antimicrobiana contra bactérias gram-positivas, gram-negativas, fungos filamentosos e leveduras, em estados planctônicos, como séssil (Naveed et al., 2019; Lima et al., 2021).

\subsection{Atividade Antimicrobiana da Quitosana}

O expressivo acréscimo nos casos de resistência bacteriana na última década, acarretada pela dificuldade de tratamentos eficazes com aplicação de antibióticos. Os empecilhos para suas medidas terapêuticas influenciam em pesquisas sobre superfícies implantes que resistem à colonização microbiana e, consequente, formação de biofilme. As estratégias empregadas para prevenir a adesão dos patógenos baseiam-se no revestimento de dispositivos médicos implantáveis com compostos que geram superfícies antiadesivas/repelentes de bactérias (polímeros), assassínios de contatos (peptídeos antimicrobianos) ou superfícies antimicrobianas de libertação (metais e biocidas). Apesar dos esforços para reduzir a incidência das infecções associadas aos implantes, majoritário dos revestimentos desenvolvidos apresenta baixa biocompatibilidade e toxicidade para as células humanas (Lima et al., 2021).

As infecções bacterianas em dispositivos médicos é um problema clínico global que ameaça tratamentos e a saúde dos pacientes, como já citado acima, muitas pesquisas são feitas com o intuito de extinguir ou minorar os riscos dessas patologias. Os pontos prioritários são precatar a adesão e proliferação bacteriana, que pretendem isolar fontes de contenção e suprimir o crescimento de potenciais colônias danosas (Kumorek et al.,2019).

A modificação química da CS ou a aglutinação dessas com reagentes fornecem soluções para esse problema, pois essas abordagens são capazes de melhorar propriedades como solubilidade e desempenho antimicrobiano do biomaterial, e as propriedades relevantes de compósitos, conseguem o seu aprimoramento (Hu et al.,2021). As diferentes proporções dos monossacarídeos constituintes da quitosana acarretam distintas atribuições físico-químicas, como peso molecular (MW), grau de desacetilação (DD) e viscosidade. Esses determinaram em grande parte as atividades antibacterianas e anti-biofilme da CS (Khan et al., 2020).

Diversos autores relataram suas atividades bactericidas ou bacteriostáticas, logo se aspira que os cátions interajam eletrostaticamente com componentes negativos (proteínas), ocasionando a inibição dos biofilmes. Dessa forma, a CS é, usualmente, aplicada na proteção de dispositivos médicos, no caso de cateteres e implantes, principalmente para MRSA, Streptococcus $\beta$-hemolítico e Pseudomonas aeruginosa (Khan et al., 2020). 
De acordo com Li et al., (2022), investigou suas composições e suas características mecânicas, confirmando seu efeito bacteriostática no $S$. aureus e bactericida na $E$. coli correlacionando com sua fibra, MW e DD, contribuindo para averiguar a eficiência em razão da sua estrutura, incentivando a empregabilidade e aperfeiçoamento da mesma. A ação bactericida contra S. aureus desestabiliza suas estruturas intracelulares e parede celular, infere-se que essa representa um potencial biopolímero como agente antibacteriano a fim de combater e auxiliar no controle da resistência ao ambiente hospitalar (Oliveira et al.,2019).

Referente à pesquisa do Costa et al., (2017), a CS demonstra um elevado potencial contra o Acinetobacter baumannii, mesmo com cepas resistentes, não interferiu na sua ação bacteriana, com a concentração mínima inibitória (MIC), de 0,5 a $1 \mathrm{mg} / \mathrm{mL}$, atuando na inibição da aderência do biofilme. Dessa forma, igualmente é utilizada nos cateteres urinários por sua atividade de reduzir ou evitar o exopolissacarideo da proliferação de Klebsiella spp. e E. coli (Campana et al., 2018).

Essa resulta na permeabilidade entre o biopolímero e nas células bacterianas e fungicas por interações eletrostáticas. Dessa forma, deve-se atribuir à sua composição química que leva à formação de um complexo policatiônico entre o grupo amino da quitosana e a membrana celular externa do agente. De um modo geral, a permeabilização da superfície celular causa o vazamento de substâncias intracelulares, que eventualmente leva a apoptose das mesmas (Jianhui Li et al., 2020; Cremar et al., 2018). A cadeia molecular da CS possui grupos funcionais (hidroxila e amino) que facilitam a formação de interações, que permitem as estruturas interconectadas dentro dos compostos por sua base. Esse contacto permitiu que membros da comunidade científica projetassem e estruturassem arquiteturas funcionais baseadas nesse biomaterial usando métodos incluindo montagem layer-by-layer (LBL) (Hu et al., 2021).

\subsection{Layer-by-layer (LBL)}

O método LBL, por definição, é uma ferramenta poderosa para preparar novos materiais através da deposição sequencial de camadas (Zhao et al., 2019). A abordagem dessa metodologia oferece controle sobre a composição, arquitetura, espessura e propriedades mecânicas do filme variando a condições de montagem. Alguns dos benefícios do método são devidos o filme poder anexar e liberar uma variedade de biomoléculas frágeis, como proteínas (DNA), enzimas e polissacarídeos, salvaguardando a atividade biológica dessas biomoléculas. Portanto, a abordagem fornece uma ferramenta simples e versátil para criar revestimentos biofuncionalizados em qualquer tipo de geometria de substrato, como superfícies de implantes médicos (Kumorek et al., 2019). Nesse contexto, o referido material reage com outros materiais através de interações, incluindo força eletrostática, ligação de hidrogênio, ligação de base de Schiff (Hu et al., 2021; Paris et al., 2021).

A CS é almejada por suas características de transfecção, gelificação in situ, inibição da bomba de efluxo, liberação controlada de drogas, mucoadesão. Além as suas propriedades atribuídas ao seu grupo funcionais amina primária e sua bioadesão que permite a aderência do biopolímeros a tecidos brandos ou rígidos, assim utilizados nas áreas de odontologia, oftalmologia, áreas de medidas ortopédicas e cirúrgicas (Ali \& Ahmed, 2018; Ashrafizadeh et al., 2021).

Ademais, com base em teorias de pesquisadores, o LBL é empregado para projetar biomateriais baseados em CS e as interações da força motriz, como propriedades biológicas dos materiais produzidos para aplicações biomédicas, amplamente reconhecidas. Por exemplo, o LBL permite a integração da funcionalidade da biocompatibilidade da CS e da atividade terapêutica de drogas e proteínas (Paris et al., 2021). Utilizando suas vantagens, estudos demonstraram a eficácia dessa técnica utilizando a CS com propriedades anti aderentes introduzidas, os revestimentos biodegradáveis restringiram a aderência de Staphylococcus aureus em cerca de 80\% dessas superfícies (Kumorek et al., 2019). 


\section{Conclusão}

Posto isso, verifica-se que uma forma provável de combater as infecções bacterianas relacionadas à ventilação mecânica é a quitosana e seus derivados, que possuem atividades antimicrobianas relevantes e grande potencial para utilização dos mesmos no combate a tenacidade de microorganismos. As múltiplas características desse biomaterial, citadas por diversos autores, reforçam a possibilidade da utilização do mesmo no combate a PAVM, interferindo na proliferação de biofilmes. A CS age através de sua composição química, que interage com a membrana celular do patógeno, levando o mesmo a realizar apoptose. Destarte, as propriedades mucoadesivas e antibacterianas do biopolímero corroboram para investigações e investimentos em cateteres da ventilação mecânica, evitando o biofilme e, com isso, reduzir a resistência bacteriana, as despesas médicas e o desconforto do enfermo.

\section{Referências}

Agência Nacional de Vigilância Sanitária (ANVISA). (2017). Critérios diagnósticos de infecção relacionada à assistência à saúde. (2a ed.), ANVISA; http://www20.anvisa.gov.br/segurancadopaciente/index.php/publicacoes/item/criterios-diagnosticos-das-infeccoes-relacionadas-a-assistencia-asaude

Ali, A. \& Ahmed, S. (2018). A review on chitosan and its nanocomposites in drug delivery. International Journal of Biological Macromolecules, 109, 273286. 10.1016/j.ijbiomac.2017.12.078

Ashrafizadeh, M. Delfi M, Hashemi F, Zabolian A, Saleki H, Bagherian M, Azami N, Farahani M. V, Sharifzadeh S. O, Hamzehlou S, Hushmandi K, Makvandi P, Zarrabi A, Hamblin M. R, \& Varma R. S. (2021).Biomedical application of chitosan-based nanoscale delivery systems: Potential usefulness in siRNA delivery for cancer therapy. Carbohydrate Polymers, 26010.1016/j.carbpol.2021.117809.

Campana, R., Biondo, F., Mastrotto, F., Baffone, W., \& Casettari, L. (2018). Chitosans as new tools against biofilms formation on the surface of silicone urinary catheters. International Journal of Biological Macromolecules. 10.1016/j.ijbiomac.2018.07.08.

Costa, E. M., Silva, S., Vicente, S., Veiga, M., Tavaria, F., \& Pintado, M. M. (2017). Chitosan as an effective inhibitor of multidrug resistant Acinetobacter baumannii. Carbohydrate Polymers, 178, 347-351. 10.1016/j.carbpol.2017.09.055

François, B., Laterre, P. F., Luyt, C. E., \& Chastre, J. (2020). The challenge of ventilator-associated pneumonia diagnosis in COVID-19 patients. Critical care (London, England), 24(1), 289. https://doi.org/10.1186/s13054-020-03013-2

Gondil, S. V., Harjai, K., \& Chhibber, S., (2021). Investigating the potential of endolysin loaded chitosan nanoparticles in the treatment of pneumococcal pneumonia. Journal of Drug Delivery Science and Technology 61, 102142, 2021.doi.org/10.1016/J.JDDST.2020.102142

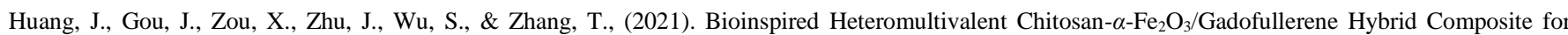
Enhanced Antibiotic-Resistant Bacterial Pneumonia.Journal of Biomedical Nanotechnology 17 (6), 1217-1228, 202. 10.1166/jbn.2021.3093

Karakuzu, Z., Iscimen, R., Akalin, H., Kelebek Girgin, N., Kahveci, F., \& Sinirtas, M. (2018). Fatores de risco prognóstico na pneumonia associada ao ventilador. Monitor de ciências médicas: revista médica internacional de pesquisa experimental e clínica,24, 13211328. https://doi.org/10.12659/msm.905919

Khan, F. Pham, D. T. N. Oloketuyi, S. F. Manivasagan, P. Oh, J. \& Kim, Y. (2020). Chitosan and their derivatives: Antibiofilm drugs against pathogenic bacteria, Colloids and Surfaces B: Biointerfaces, Volume 185,110627. 10.1016/j.colsurfb.2019.110627.

Kucukoglu, V., Uzuner H., Kenar H., \& Karadenizli A., (2019). In vitro antibacterial activity of ciprofloxacin loaded chitosan microparticles and their effects on human lung epithelial cells. International Journal of Pharmaceutics 569, 118578, 2019. 10.1016/J.IJPHARM.2019.118578

Lamiyan, A. K., Dalal, R., \& Kumar, N. R. (2020). Venom peptides in association with standard drugs: a novel strategy for combating antibiotic resistance-an overview. Journal of Venomous Animals and Toxins including Tropical Diseases, 26. 10.1590/1678-9199-JVATITD-2020-0001

Li, J Fu, J. Tian, X. Hua, T. Poon, T. Koo, M \& Chan, W. (2022).Characteristics of chitosan fiber and their effects towards improvement of antibacterial activity, Carbohydrate Polymers, Volume 280. 10.1016/j.carbpol.2021.119031.

Lima, J. L. D. C., Alves, L. R., Paz, J. N. P. D., Rabelo, M. A., Maciel, M. A. V., \& Morais, M. M. C. D. (2017). Analysis of biofilm production by clinical isolates of Pseudomonas aeruginosa from patients with ventilator-associated pneumonia. Revista Brasileira de Terapia Intensiva, 29, 310-316.. 10.5935/0103507X.20170039

Madni, A., Kousar, R., Naeem, N., \& Wahid, F. (2021). Recent advancements in applications of chitosan-based biomaterials for skin tissue engineering. Journal of Bioresources and Bioproducts, 6(1), 11-25. 10.1016/j.jobab.2021.01.002

Mohan, K., Muralisankar, T., Jayakumar, R., \& Rajeevgandhi, C. (2021). A study on structural comparisons of $\alpha$-chitin extracted from marine crustacean shell waste. Carbohydrate Polymer Technologies and Applications, 2, 100037. 10.1016/j.carpta.2021.100037

Mohd Sazlly, L. S., Zainal Abidin, A., Liew, S., Roberts, J., \& Sime, F. (2019). The global prevalence of multidrug-resistance among Acinetobacter baumannii causing hospital-acquired and ventilator-associated pneumonia and its associated mortality: A systematic review and meta-analysis. Journal of Infection. 10.1016/j.jinf.2019.09.012 
Naveed, M., Phil, L., Sohail, M., Hasnat, M., Baig, M. M. F. A., Ihsan, A. U., \& Zhou, Q. G. (2019). Chitosan oligosaccharide (COS): An overview. International journal of biological macromolecules, 129, 827-843. 10.1016/j.ijbiomac.2019.01.192

Organização Mundial da Saúde. Nomear a doença coronavírus (COVID-19) $e \quad$ o vírus que a causa. (2020). em: https://www.who.int/emergencies/diseases/novel-coronavirus-2019/technical-guidance/naming-the-coronavirus-disease-(covid-2019)-and-the-virus-thatcauses

Papazian, L. Klompas, M. \& Luyt, C. E. (2020). Ventilator-associated pneumonia in adults: a narrative review. Intensive Care Medicine, 46 (5), 888906. 10.1007/s00134-020-05980-0

Pinto, A. C. D. S., Silva, B. M. D., Santiago-Junior, J. F., \& Sales-Peres, S. H. D. C. (2021). Efficiency of different protocols for oral hygiene combined with the use of chlorhexidine in the prevention of ventilator-associated pneumonia. Jornal Brasileiro de Pneumologia, 47. 10.36416/1806-3756/e20190286

Santos, C. D., Nascimento, E. R. P. D., Hermida, P. M. V., Silva, T. G. D., Galetto, S. G. D. S., Silva, N. J. C. D., \& Salum, N. C. (2020). Good nursing practices towards patients on invasive mechanical ventilation in hospital emergencya. Escola Anna Nery, 24. 10.1590/2177-9465-EAN-2019-0300

Sazlly, L. S. M. Abidin, A. Z. Liew, S. Roberts, J. \& Sime, F. (2019). The global prevalence of multidrug-resistance among Acinetobacter baumannii causing hospital-acquired and ventilator-associated pneumonia and its associated mortality: A systematic review and meta-analysis. Journal of Infection. 10.1016/j.jinf.2019.09.012

Shannon, M. F. Tran, A. Cheng, W. Klompas, M. Kyeremanteng, K. Mehta, S. English, S. W., Muscedere, J. Cook, D. J. Torres, A. Ranzani, O. T. FoxRobichaud, A E. Alhazzani, W. Munshi, L. Guyatt, G. .H, \& Rochwerg, B. (2020). Diagnosis of ventilator-associated pneumonia in critically ill adult patientsa systematic review and meta-analysis. Intensive Care Med.46 (6), 1170-1179. 10.1007/s00134-020-06036-z

Sidrim, J. J., Amando, B. R., Gomes, F. I., do Amaral, M. S., de Sousa, P. C., Ocadaque, C. J., \& SCM Castelo-Branco, D. D. (2019). Chlorpromazineimpregnated catheters as a potential strategy to control biofilm-associated urinary tract infections. Future Microbiology, 14(12), 1023-1034. 10.2217/fmb2019-0092

Silva, D. H. F., Camargos, J. H. D., Rodrigues, J. G., Nogueira, L. S., Azevedo, D. A. D., Carvalho, M. D. G., \& Pinheiro, M. D. B. (2020). Impact of oral hygiene in patients undergoing mechanical ventilation in the COVID-19 pandemic. Revista da Associação Médica Brasileira, 66, 96-101. 10.1590/18069282.66.S2.96

Silva, P., Paranhos, LR, Meneses-Santos, D., Blumenberg, C., Macedo, D. R, \& Cardoso, S. V (2021). Combinação de escovação dentária e clorexidina em comparação com o uso exclusivo de clorexidina para reduzir o risco de pneumonia associada à ventilação: Uma revisão sistemática com meta-análise. Clinics (São Paulo, Brasil) , 76, e2659. 10.6061/clinics/2021/e2659

Soussan, R., Schimpf, C., Pilmis, B., Degroote, T., Tran, M., Bruel, C., \& Philippart, F. (2018). Ventilator-associated pneumonia: The central role of transcolonization. Journal of Critical Care. 10.1016/j.jcrc.2018.12.005

Souza, L. C. D., Mota, V. B. R. D., Carvalho, A. V. D. S. Z. D., Corrêa, R. D. G. C. F., Liberio, S. A., \& Lopes, F. F. (2017). Association between pathogens from tracheal aspirate and oral biofilm of patients on mechanical ventilation. Brazilian oral research, 31. 10.1590/1807-3107BOR-2017.vol31.0038

Suaya, J. A., Fletcher, M. A., Georgalis, L., Arguedas, A., McLaughlin, J. M., Ferreira, G., \& Verstraeten, T. (2020). Identification of Streptococcus pneumoniae in Hospital-acquired Pneumonia in Adults: A Systematic Review. Journal of Hospital Infection. 10.1016/j.jhin.2020.09.036

Vazquez Guillamet, C., \& Kollef, M. H. (2018). Is Zero Ventilator-Associated Pneumonia Achievable? Clinics in Chest Medicine, 39(4), 809-822. 10.1016/j.ccm.2018.08.004

Tan, T. N., Thi, T. H. N., San-Lang, W., Thi, P. K. V., \& Anh, D. N., (2017). Preparation of chitosan nanoparticles by TPP ionic gelationcombined with spray drying, and the antibacterial activity of chitosan nanoparticles and a chitosan nanoparticle-amoxicillin complex. Res Chem Intermed. 43:3527-3537 $10.1007 / \mathrm{s} 11164-016-2428-8$

Wu D, Wu C, \&Zhang S, \& Zhong Y. (2019). Fatores de risco de pneumonia associada ao ventilador em pacientes criticamente III. Front Pharmacol. 10:482.10.3389/fphar.2019.00482. 